\title{
Craters on silicon surfaces created by gas cluster ion impacts
}

\author{
L. P. Allen ${ }^{\text {a) }}$ \\ Epion Corporation, 37 Manning Road, Billerica, Massachusetts 01821 \\ Z. Insepov \\ Himeji Institute of Technology, Laboratory of Advanced Science and Technology for Industry, 3-1-2 Kouto, \\ Kamigori, Ako, Hyogo 678-1205 Japan \\ D. B. Fenner, C. Santeufemio, and W. Brooks \\ Epion Corporation, 37 Manning Road, Billerica, Massachusetts 01821 \\ K. S. Jones \\ Department of Materials Science and Engineering, University of Florida, Gainesville, Florida 32611 \\ I. Yamada \\ Himeji Institute of Technology, Laboratory of Advanced Science and Technology for Industry, 3-1-2 Kouto, \\ Kamigori, Ako, Hyogo 678-1205 Japan
}

(Received 4 February 2002; accepted for publication 16 July 2002)

\begin{abstract}
Atomic force microscopy (AFM) and high-resolution transmission electron microscope (HRTEM) cross section imaging of individual gas cluster ion impact craters on $\mathrm{Si}(100)$ and $\mathrm{Si}(111)$ substrate surfaces is examined. The comparison between 3 and $24 \mathrm{kV}$ cluster impacts from $\mathrm{Ar}$ and $\mathrm{O}_{2}$ gas sources is shown. Results for low fluence $\left(10^{10} \mathrm{ions} / \mathrm{cm}^{2}\right) 24 \mathrm{kV}$ Ar individual cluster impacts onto a $\mathrm{Si}(100)$ and $\mathrm{Si}(111)$ substrate surfaces are compared with hybrid molecular dynamics (HMD) simulations. A HMD method is used for modeling impacts of $\operatorname{Ar}_{n}(n=135,225)$ clusters, with energies of 24-50 eV/atom, on $\mathrm{Si}(100)$ and $\mathrm{Si}(111)$ surfaces. On a $\mathrm{Si}(100)$, craters are nearly triangular in cross section, with the facets directed along the close-packed (111) planes. The $\mathrm{Si}(100)$ craters exhibit four-fold symmetry as imaged by cross-sectional HRTEM, and AFM top view, in agreement with modeling. In contrast, the shape of craters on a $\mathrm{Si}(111)$ shows a complicated six-pointed shape in the modeling, while AFM indicates three-fold symmetry of the impact. The lower energy $3 \mathrm{kV}$ individual cluster impacts reveal the same crater shape in HRTEM cross section for both $\mathrm{Ar}$ and $\mathrm{O}_{2}$ gas clusters, but with shallower crater depth than for the higher-energy impacts. The kinetics of the $\mathrm{Ar}$ and $\mathrm{O}_{2}$ crater impacts may explain the successful use of higher-energy cluster impacts for etching material of higher initial surface roughness followed by the lower-energy impacts as an effective finishing step to achieve smoother surfaces. (C) 2002 American Institute of Physics. [DOI: 10.1063/1.1506422]
\end{abstract}

\section{INTRODUCTION}

Interactions of energetic clusters of atoms with solid surfaces demonstrate unique phenomena and promise new applications for surface modification technology. ${ }^{1-7}$ Clusters of gaseous elements and compounds consisting of hundreds to thousands of atoms, with energies from a few $\mathrm{eV}$ to a few hundreds of eV per cluster atom are of particular interest for surface modification.

The main surface modification phenomenon for gas cluster ion beam (GCIB) technology is the surface smoothing effect. A crystal surface, with an initial average surface roughness of tens or hundreds of angstroms, becomes atomically flat, with the residual roughness of a few angstroms. ${ }^{5,8-13}$ The GCIB smoothing typically occurs after irradiation by an ionized $\operatorname{Ar}_{n}(n \sim 1000-10000)$ cluster beam at a charge fluence of $10^{14}-10^{16}$ ions $/ \mathrm{cm}^{2}$.

The so-called "lateral sputtering effect" that was predicted by molecular dynamics (MD) simulation and verified

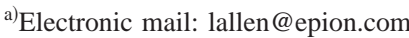

experimentally by the Kyoto group ${ }^{9,10}$ is used to explain the surface smoothing effect that is observed for metal, semiconductor, and insulating surfaces. According to the lateral sputtering effect, smoothing occurs as a cooperative result of multiple atomic-scale "bursts" caused by energetic cluster ion impacts onto surfaces. Most of the surface material involved in such bursts does not leave the surface at all but becomes highly mobile, and therefore diffuses quickly along the lateral direction, thus making the surface smoother.

An individual cluster impact would leave a crater on the surface, but it is impossible to experimentally study those craters at a high cluster ion fluence that is typical for surface smoothing processes. The study of the crater formation, crater structure, and faceting properties with low cluster ion fluence are important tasks for understanding the fundamental surface science and smoothing effect.

The kinetics of an atomic cluster ion impinging on a surface is quite different from that of an ion implanted atom into a target. A 1000 atom gas cluster accelerated through 10 $\mathrm{kV}$ yields an average individual atom energy of $10 \mathrm{eV}$ as the cluster impacts the surface. This average energy is very large 


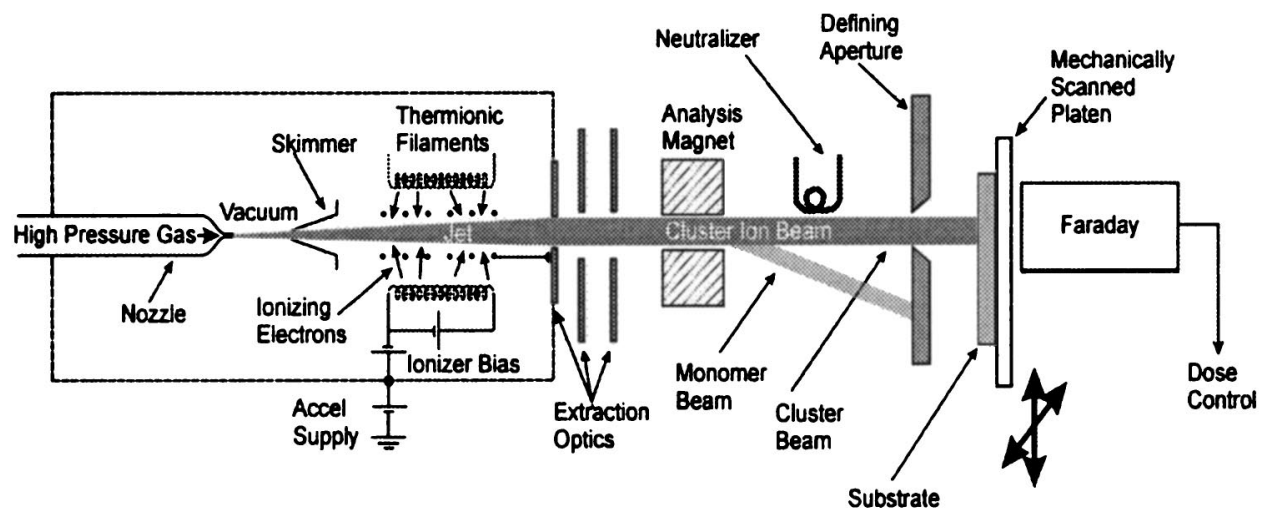

FIG. 1. A gas cluster ion-beam apparatus schematic.

compared with the typical binding energy $(<0.1 \mathrm{eV} /$ argon atom) of the clusters, and hence the impacts are highly inelastic.

While experimental data are still sparse, ${ }^{1,2,4,5}$ modeling and postatomic cluster impact analysis may help to evaluate the physical nature and mechanisms involved in the gas cluster ion-beam process. The aim of this work is to study individual $\mathrm{Ar}$ gas cluster impact data obtained by high-resolution transmission electron microscopy (HRTEM), atomic force microscopy (AFM), and MD simulations. High- and lowenergy $\mathrm{Ar}$ and $\mathrm{O}_{2}$ gas cluster impacts are observed in cross section by HRTEM. AFM is used to examine the crater surface shapes for the high-energy Ar cluster impacts for two different silicon surface orientations. The cross section and surface shape of the high-energy Ar gas cluster impact craters are compared directly with MD simulations.

\section{EXPERIMENT}

The general GCIB apparatus schematic is shown in Fig. 1. Gas cluster formation is achieved through supersonic gas expansion from a high-pressure source into a vacuum via a small orifice or nozzle. The adiabatic expansion reduces the relative velocity of the gas atoms and condensation into clusters is favored. The nozzle, a key component to gas cluster beam formation, determines the cluster size and beam flow pattern and will serve to remove some heat from the atomic collision process, thus assisting in the gas cluster formation. Clusters with mean sizes anywhere from 500 to 10000 atoms of a source gas can be held together by van der Waals forces. A small aperture, or skimmer, collects the primary jet core of gas clusters. The forward-directed neutral clusters are ionized by impact of electrons emitted and accelerated from a filament (Fig. 1). This forms positive ion gas clusters of nominally one electron charge per cluster. The ionized clusters are extracted and accelerated (typically from 2 to $25 \mathrm{kV}$ ) using a series of electrodes that acts as a mass filter. Applying a low voltage on the first extraction electrode ensures that only clusters of large kinetic energies are further accelerated. Electrostatic lenses are utilized to focus the cluster ions, and monomers are filtered out through the use of a transverse magnetic field. Ion fluence is measured by a Faraday cup, and in the system shown next, the sample is mechanically scanned for complete wafer coverage.
In this study, polished silicon substrates with a native oxide were exposed to low fluence $\left(10^{10}\right.$ ions $\left./ \mathrm{cm}^{2}\right)$ argon and $\mathrm{O}_{2}$ gas cluster ions. HRTEM cross section images of the individual cluster impacts for $\mathrm{Ar}$ and $\mathrm{O}_{2}$ at 3 and $24 \mathrm{kV}$ acceleration energies were obtained. AFM images of the larger $24 \mathrm{kV}$ Ar cluster impact craters were analyzed. HRTEM revealed that no dislocation formation or lattice amorphization occurs in the near surface or subsurface regions. The GCIB smoothing process may be envisioned as a stochastic overlay of individual shallow craters. The respective shallow crater shapes of the $3 \mathrm{kV}$ Ar and $3 \mathrm{kV} \mathrm{O}_{2}$ cluster impacts appear to provide a fundamental basis for the resulting smoothness of material surfaces.

\section{MODEL OF INDIVIDUAL GAS CLUSTER ION IMPACT}

\section{A. Simulation model}

In a MD simulation, the equations of motion of interacting particles are solved numerically. Appropriate initial and boundary conditions are supplied. Due to limited computing resources, there is always a problem of choosing a correct size for the model system. The number of mobile atoms chosen will set up the MD model size: Too few atoms may show unphysical effects but increasing it has a steep price in computation time and required memory as both grow rapidly with an increasing number of the degrees of freedom of the system. The usual procedure is to estimate a necessary number of the target atoms, often influenced by the available computing power, and to account for the rest of the system by proper boundaries which would allow the flow of energy deposited by the impact to the bulk of the solid. One of the often-used techniques, based on stochastic Langévin dynamics, employs two or three atomic layers surrounding the central volume treated by MD. These layers of damped atoms represent a "thermal bath" which simulates heat transfer to the bulk of the target. This technique seems to be reasonable in simulation of low-energy processes, such as film deposition.

Energetic cluster impacts create violent collisions between atoms in the central zone where equivalent temperature and pressure may reach $10^{5} \mathrm{~K}$ and $10^{6}$ bars, respectively. ${ }^{5}$ For modeling of such events, the boundary can be made "flexible" by allowing its expansion to keep the 
average pressure constant for a given modulus of elasticity. This technique is not completely satisfactory as it uses the average pressure, which depends on the system size, and requires the knowledge of materials characteristics, such as thermal conductivity and compressibility. These parameters cannot be reliably extrapolated from the normal equilibrium conditions to the extreme state of matter in the collision zone.

The problem of the boundary conditions can be also examined by considering shock waves created by the energetic cluster impact. Unphysical reflections of the shock waves from the system boundary may show up in MD results, distorting the picture of the investigated process. Shock wave reflections have been revealed for the systems as large as 4 $\times 10^{5}$ target atoms studied by MD if fixed periodic boundary conditions are used, for the cluster impact energy as low as $10 \mathrm{kV} .^{14,15}$

A new hybrid model utilizing MD for the atoms in the central collision zone, and continuum mechanics and thermodynamics outside, has been proposed in a previous paper $^{16}$ and used for crater formation study in Ref. 17. In this model, a shock wave theory is used to establish the minimum size of the central zone, i.e., the location of the boundary between the volume where MD models atomic collisions and the outside, which is treated as continuum. This hybrid model successfully solves the problem related to a finite system size by reducing the number of the required degrees of freedom, and will be applied to the modeling of craters in the present article. For convenience, some equations of the hybrid model are given next.

According to the shock wave theory, ${ }^{18}$ the distance $L$ from the center of the cluster impact to where the shock wave becomes thermally equilibrated can be estimated as $L$ $\approx d \times 10^{1 / 3}$, where $d$ is the cluster diameter. For argon clusters with 100 atoms, $d \approx 30 \AA$ and $L$ is of the order of $100 \AA$. The shock wave travels distance $L$ in time $\tau_{0}=L / \nu_{0}$, where $\nu_{0}$ is the cluster velocity (prior to impact, i.e., an upper bound). For argon clusters with kinetic energy of $20 \mathrm{eV}$ per atom, $\nu_{0} \sim 10^{4} \mathrm{~m} / \mathrm{s}$, and the time interval is: $\tau_{0} \sim 1 \mathrm{ps}$.

The hybrid model establishes the boundary of MD volume at $L$ and follows the system evolution with MD calculations for the time at least several times $\tau_{0}$. The volume beyond radius $L$ from the point of impact is treated as a continuum, using a finite-element method to solve continuum mechanics equations

$$
\rho \frac{d^{2} \mathbf{u}_{i}}{d t^{2}}=\frac{\partial \sigma_{i k}}{\partial x_{k}}
$$

where $u_{j}$ is the displacement vector of the $j$ th cell, $\sigma_{i k}$ is the stress tensor, and $\rho$ is the solid density. ${ }^{19}$

Heat transfer is described by

$$
\frac{d T(\mathbf{r}, t)}{d t}=\chi \Delta T(\mathbf{r}, t)
$$

with temperature (T) at position $r$ and time $t$. Equations (1a) and (1b) are coupled by the stress tensor dependence on material constants and temperature:

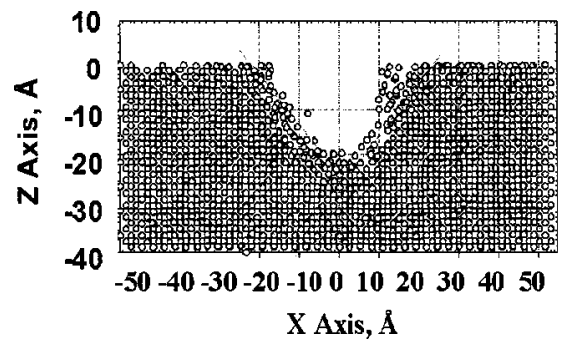

FIG. 2. Side view of the crater formed by a $\mathrm{Ar}_{135}$ cluster impact, with energy of $24 \mathrm{eV} /$ atom, on a $\mathrm{Si}(100)$ surface after $14.4 \mathrm{ps}$.

$$
\begin{aligned}
\sigma_{i k}= & -K \alpha T \delta_{i k}+K u_{l l} \delta_{i k}+2 \mu\left(u_{i k}-u_{l l} \frac{\delta_{i k}}{3}\right)+\xi \dot{u}_{l l} \delta_{i k} \\
& +2 \eta\left(\dot{u}_{i k}-\dot{u}_{l l} \frac{\delta_{i k}}{3}\right) \\
u_{i k}= & \frac{1}{2}\left(\frac{\partial u_{i}}{\partial x_{k}}+\frac{\partial u_{k}}{\partial x_{i}}\right),
\end{aligned}
$$

where $\alpha$ is the thermal expansion coefficient, $K$ and $\xi$ the bulk elastic modulus and viscosity, $\mu$ and $\eta$ are the sheer modulus and viscosity, and $u_{i k}$ is the strain tensor.

For silicon at room temperature, $\chi \sim 1 \mathrm{~cm}^{2} / \mathrm{s},{ }^{20}$ so that the characteristic heat transfer time $\tau_{1}=L^{2} / \chi=1 \mathrm{ps}$ is similar to the characteristic deformation time $\tau_{0}$. This is convenient for numerical computation as it allows the use of common time steps for calculating thermal and mechanical variables. Equations (1a)-(1d) have been used to numerically solve a two-dimensional problem projected by cylindrical geometry defined by the symmetry of the system.

In the MD calculations, we used the Buckingham potential to represent two-body forces between cluster atoms and between the cluster and target atoms while interactions between $\mathrm{Si}$ atoms were represented by the Stillinger-Weber potential. The cluster was modeled by cutting a spherical volume from a face-centered-cubic argon lattice with initial temperature set to zero.

The clusters used in simulations contained about 100200 atoms and had kinetic energy of a few tens of kV. The cylindrical target model contained $\sim 10^{5}$ atoms in the central MD zone, while the continuum mechanics calculations extended to ten times larger volume. In fact, there is no limitation for the system size in this method at all.

\section{B. Molecular dynamic results}

MD calculations give positions and momenta of all cluster atoms and the target atoms in the central zone, which provide a wealth of information on the collision process and allow us to obtain a number of parameters of interest. Figure 2 shows a side view of the simulated crater formed by a 24 eV/atom $\operatorname{Ar}_{135}$ cluster impact on a $\mathrm{Si}(100)$ surface after a time interval of $14.4 \mathrm{ps}$ from the start of cluster collision with the target. This picture shows a nearly triangular faceting of the crater, which is due to a higher energy of the (111) plane. The two dashed lines in Fig. 2 show the directions of two (111) planes. 


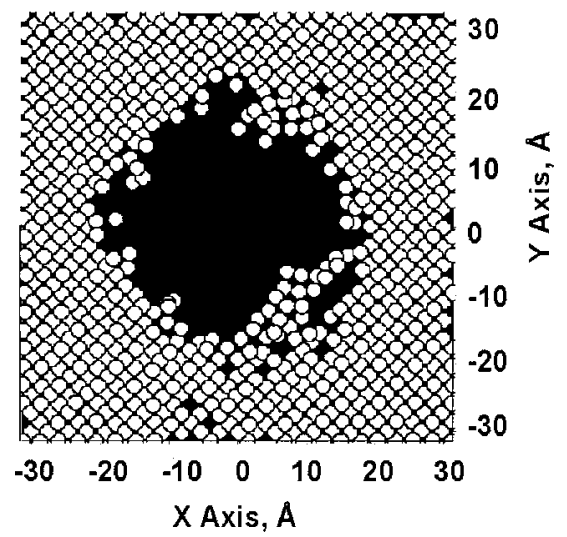

FIG. 3. Top view of the $\mathrm{Si}(100)$ surface showing the crater that was created by a $\mathrm{Ar}_{135}$ cluster impact with an energy of $24 \mathrm{eV} /$ atom.

The top view of the crater is shown in Fig. 3. It shows a four-fold symmetry crater with facets formed by four (111) planes crossing the (100) surface. In Fig. 3, only those atomic positions are shown that lie below the previous unirradiated surface. Figure 3 shows a thin slice of the sample parallel to surface that was made by cutting out the atoms with the positions within the interval: $-3 \AA<z_{i}<0.05 \AA$, where $z_{i}$ is a $\mathrm{Si}$ atomic position coordinate normal to the surface. The $\mathrm{Si}(111)$ surface shows faceting features quite different from that of the $\mathrm{Si}(100)$ surface as can be viewed in Figs. 4 and 5. We see that the side view (Fig. 4) has a roundshaped crater and the top view is also quite interesting. It has a six-point star shape, previously unobserved in simulation or in experiment. Figure 5, a top view for $\mathrm{Si}(111)$, shows a thin slice of the sample that contains four $\mathrm{Si}$ atomic layers parallel to surface, with $-4 \AA<z_{i}<0.05 \AA$.

The effect is very different from monomer ion irradiation and is clearly related to different dynamics of collisions of the two projectiles with a solid. Cluster impact results in transfer of kinetic energy to a large number of surface atoms, which leads to their displacement and local melting or sublimation.

\section{IMAGING OF CRATER IMPACTS}

\section{A. High resolution transmission electron microscopy, atomic force microscopy}

The images of individual gas cluster ion impacts were obtained using a JEOL 2010 HRTEM with a field-emission gun. Standard gluing, dimpling, and ion milling cross-

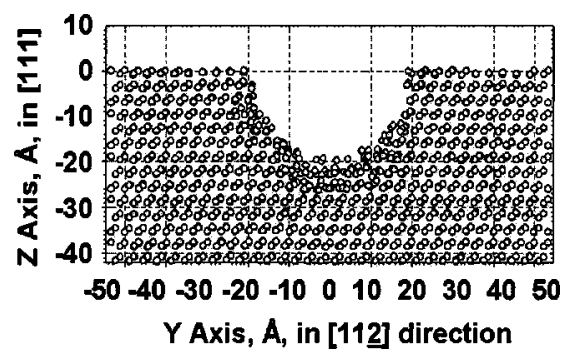

FIG. 4. Side view of the crater formed by an $\mathrm{Ar}_{135}, 24 \mathrm{eV} /$ atom, cluster impact on a $\mathrm{Si}(111)$ surface.

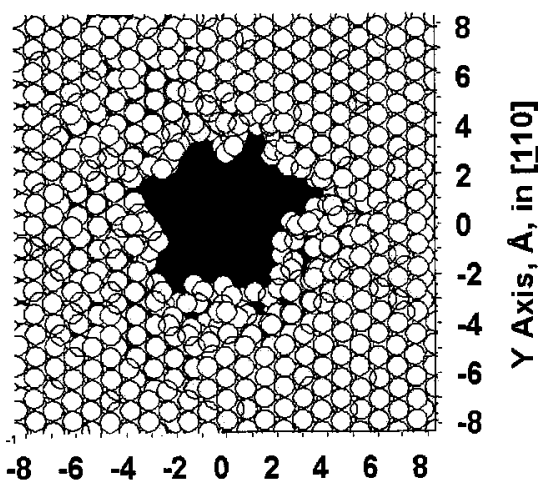

$X$ Axis, $A$, in [112] direction

FIG. 5. Top view of the $\mathrm{Si}(111)$ surface showing a crater formed by an $\mathrm{Ar}_{135}$ cluster impact with $24 \mathrm{eV} /$ atom energy.

sectional TEM sample preparation techniques were employed. Images were formed by orientation of the sample such that the transmitted beam was parallel to the $\langle 110\rangle$ direction of the lattice and parallel to the (100) plane of the surface. An objective aperture that allows transmission of 13 beams was used to form the phase contrast images.

Figure 6 shows HRTEM cross section of an individual Ar gas cluster ion crater formed by a $24 \mathrm{kV}$ acceleration into a $\mathrm{Si}(100)$ surface. The image cross section shape agrees well with Fig. 2, showing a conical impact crater with the (111) planes aligned with the crater walls. No subsurface dislocations are observed around the crater formed by the Ar gas cluster impact. The lattice integrity and orientation is preserved to the crater boundary and the surface.

Figure 7 shows HRTEM cross section of a lower-energy $3 \mathrm{kV}$ individual Ar gas cluster ion crater on a $\mathrm{Si}(100)$ surface. The shape and atomic plane cross section boundaries appear identical to that of the $24 \mathrm{kV}$ impact crater, but with a far shallower depth. In this cross section, a thin film of silicon crystal is imaged as intact over the impact crater. Such a thin film slice of silicon is envisioned for a cross section image that is taken near but not exactly at the crater center. The material is thin enough to observe the back wall of the

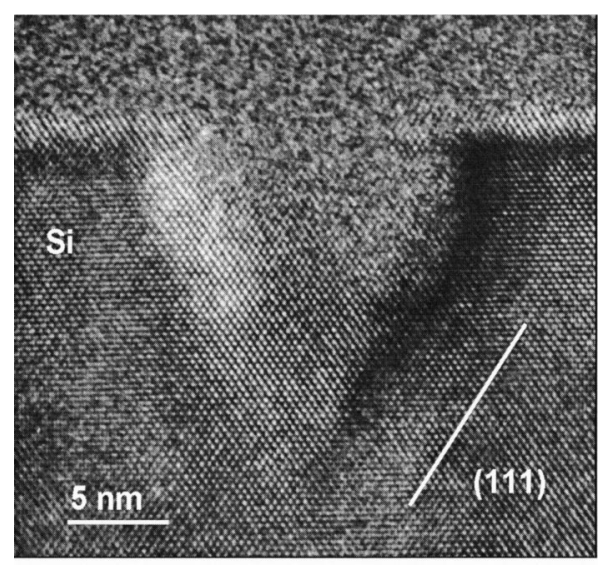

FIG. 6. HRTEM cross section image of an individual $24 \mathrm{kV}$ Ar gas cluster ion impact into $\mathrm{Si}(100)$. Crater conical edges align along the (111) lattice planes. 


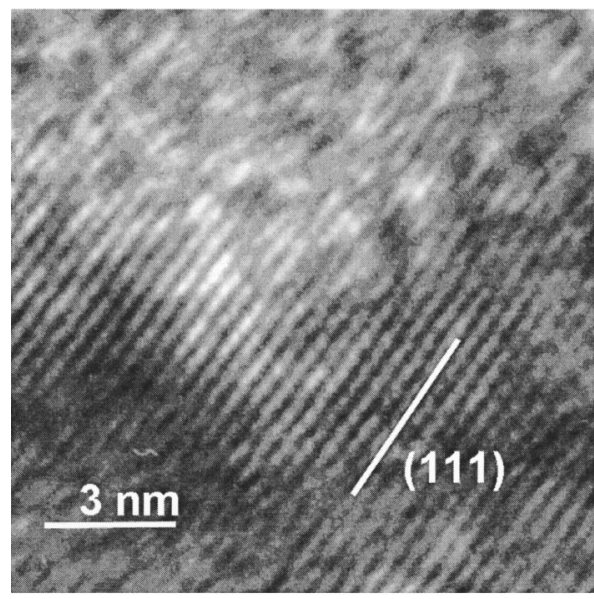

FIG. 7. HRTEM cross section image of an individual $3 \mathrm{kV}$ Ar gas cluster ion impact into $\mathrm{Si}(100)$. Crater conical edges align along the (111) lattice planes, but the depth of impact is reduced.

crater, with the outline of the full crater cross section observed.

The impact kinetics plays a first-order role in the GCIB to surface interaction, as observed by crater depth as a function of energy. This was again observed through the $24 \mathrm{kV}$ and $3 \mathrm{kV}$ individual impact crater images of an $\mathrm{O}_{2}$ cluster into silicon as shown in Figs. 8 and 9, respectively. Once again, the general shape of the impact crater is maintained in the higher- and lower-energy processes, with the lower energy impact having a shallower crater depth. In our observations, both the higher-energy and lower-energy $\mathrm{O}_{2}$ gas cluster impacts typically result in a more rounded crater shape as compared with that of the Ar impact crater. This may be due to an inherently different cluster mass, charge, or an immediate silicon oxidation that, in turn, changes the bond strength that the remaining atoms of the impinging cluster

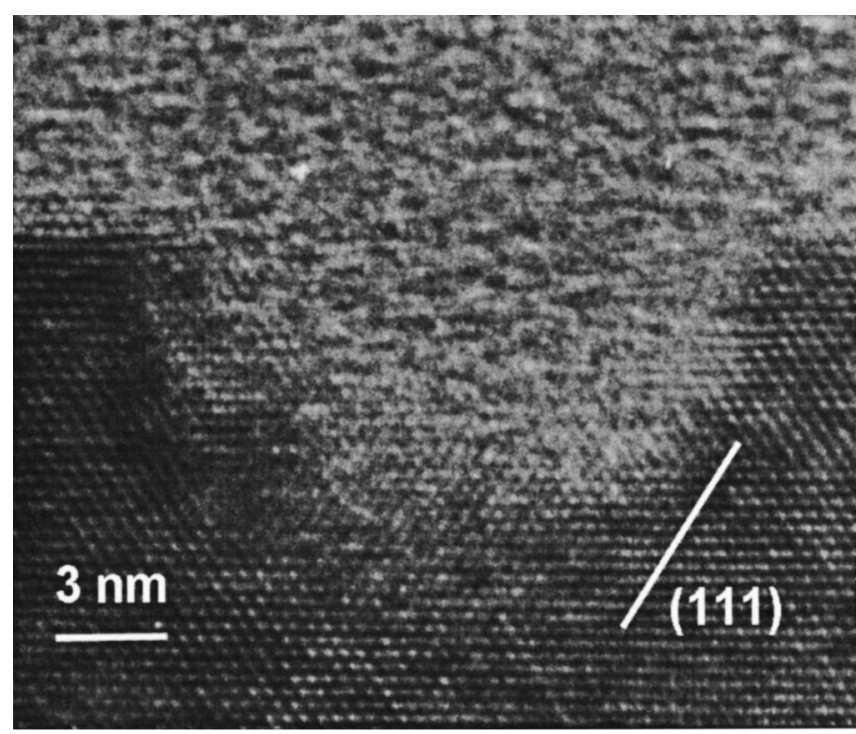

FIG. 8. HRTEM cross section image of an individual $24 \mathrm{kV} \mathrm{O}_{2}$ gas cluster ion impact into $\mathrm{Si}(100)$. Rounder impact craters typically resulted from the $\mathrm{O}_{2}$ cluster process, perhaps from the immediate oxidation of the $\mathrm{Si}$ and sidewall restructuring.

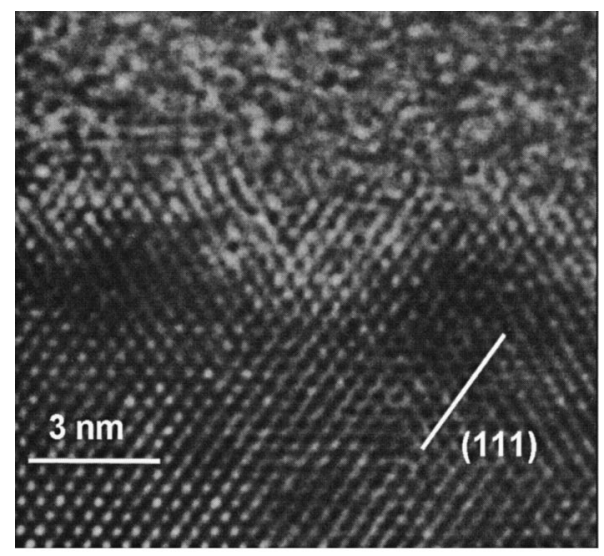

FIG. 9. HRTEM cross section of an individual $3 \mathrm{kV} \mathrm{O}_{2}$ gas cluster impact into $\mathrm{Si}(100)$. The rounder shape of the impact is preserved with the shallow depth of the crater.

"see" or will impact. No subsurface dislocations are observed around the crater formed by the $\mathrm{O}_{2}$ gas cluster impact. As in the case for Ar gas cluster impacts, the lattice integrity is preserved. The factor of chemistry in GCIB processing is a topic of significant interest and recent publication. ${ }^{2}$

\section{B. Atomic force microscopy}

In order to examine the impact crater morphology as compared with that of MD modeling, the surface of the GCIB processed silicon was also imaged by AFM. Prior to the individual cluster impacts into the silicon surface, the $\mathrm{Si}$ substrates exhibited $\sim 2 \mathrm{~nm}$ of a native oxide as measured by spectroscopic ellipsometry (SE). Upon impact by the Ar gas cluster, the silicon from the substrate lattice (as well as the native oxide) is ejected or vaporized. ${ }^{2}$ AFM imaging reveals hillock formation around and above the impact craters. The larger hillocks have been found to reflect the symmetry of the underlying $\mathrm{Si}$ substrate orientation. Figure 10 shows an example of the result of the $24 \mathrm{kV}$ Ar-GCIB individual impacts into Si surfaces with (100) and (111) substrate orientations. In the AFM image of Fig. 10(a), the symmetry of the Si substrate crystal is reflected by the distinct four-fold hillock formed by a $24 \mathrm{kV}$ Ar gas cluster impact into the $\mathrm{Si}(100)$ surface. In the AFM image of Fig. 10(b), a three-fold symmetry can be observed in the hillock formed by a $24 \mathrm{kV} \mathrm{Ar}$ gas cluster impact into the $\mathrm{Si}(111)$ surface. The image axes are aligned with the substrate crystallographic directions, as shown. AFM images indicate that the larger hillocks are $\sim 4$ $\mathrm{nm}$ high, projecting above the native oxide, with a $\sim 40 \mathrm{~nm}$ diameter. Smaller hillocks are numerous, without the clarity of symmetry found in the larger crater/hillock formations. The larger hillocks show distinct dimples in the center that correspond to the pits imaged by TEM. For low fluence, high-energy Ar cluster impacts, AFM has measured a continuum of individual hillock heights. They range from just over surface oxide height $(\sim 20 \AA)$ to over $50 \AA$ high.

\section{DISCUSSION}

High-resolution microscopic imaging by TEM and AFM of individual gas cluster impact craters for both $\mathrm{Ar}$ and $\mathrm{O}_{2}$ 

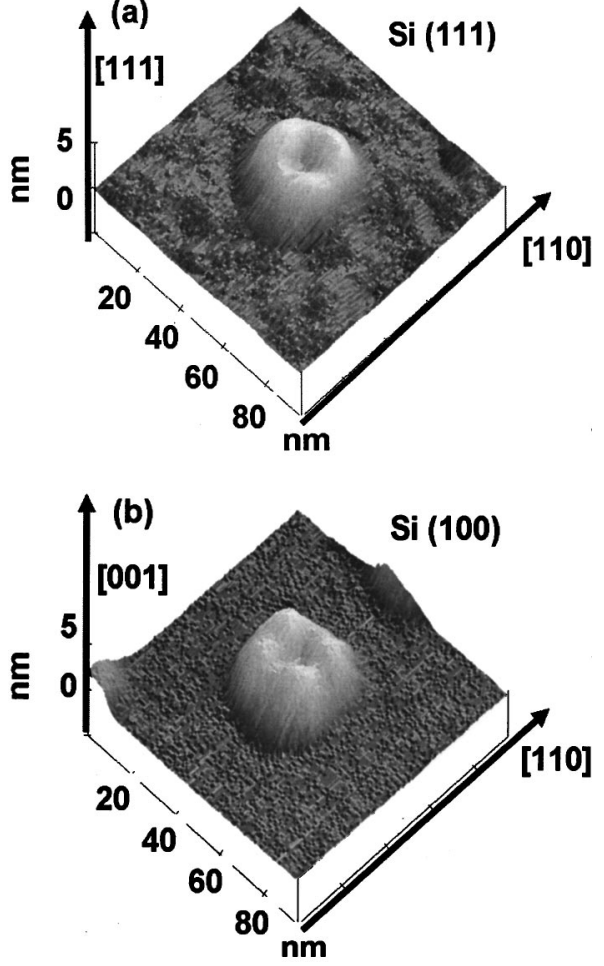

FIG. 10. Three-dimensional $100 \mathrm{~nm} \times 100 \mathrm{~nm}(z=10 \mathrm{~nm})$ AFM image of four-fold symmetry of $\mathrm{Si}(100) 24 \mathrm{kV}$ Ar cluster impact hillock (a) and three-fold symmetry of $\mathrm{Si}(111) 24 \mathrm{kV}$ Ar cluster impact hillock (b).

are analyzed. For a normal incidence angle of impact into $\mathrm{Si}(100)$, highly symmetric craters are observed. The images show the symmetric subsurface crater with crystallographic orientation along the (111) in the subsurface silicon. The impact crater shape as shown by HRTEM is in good agreement with the MD simulation for the $24 \mathrm{kV}$ Ar gas cluster case. While individual atoms are shown in some disarray, the general crater shape is consistent with (111) sidewall orientation and a four-fold symmetric crater opening.

In the AFM study, a wide distribution of individual crater sizes was observed. This is consistent with the bellshaped distribution of the mass-to-charge ratio for individual clusters that have been measured using time of flight, ${ }^{21}$ and the size distribution extending from about 1000 atoms per cluster to over 10000 atoms per cluster. The structure of such clusters has been reported as poly-icosahedral with a diameter of approximately $4.8 \mathrm{~nm}$ for a cluster of 1500 argon atoms. ${ }^{22,23}$ For each cluster impact, the symmetry and relative depth of the crater may be inferred from the shape of the hillock that forms over the crater. In the AFM images presented, the hillock that forms above craters of the $\mathrm{Si}(100)$ surface exhibits the four-fold symmetry as predicted by the MD simulation and consideration of the Si spatial density. For the case of $\mathrm{Si}(111)$, AFM imaging indicates a three-fold symmetric hillock that forms above the crater. While MD simulation predicts a six-fold symmetry, that configuration may be reduced in application by the overall three-fold hillock symmetry which covers the detail of the crater surface edge.
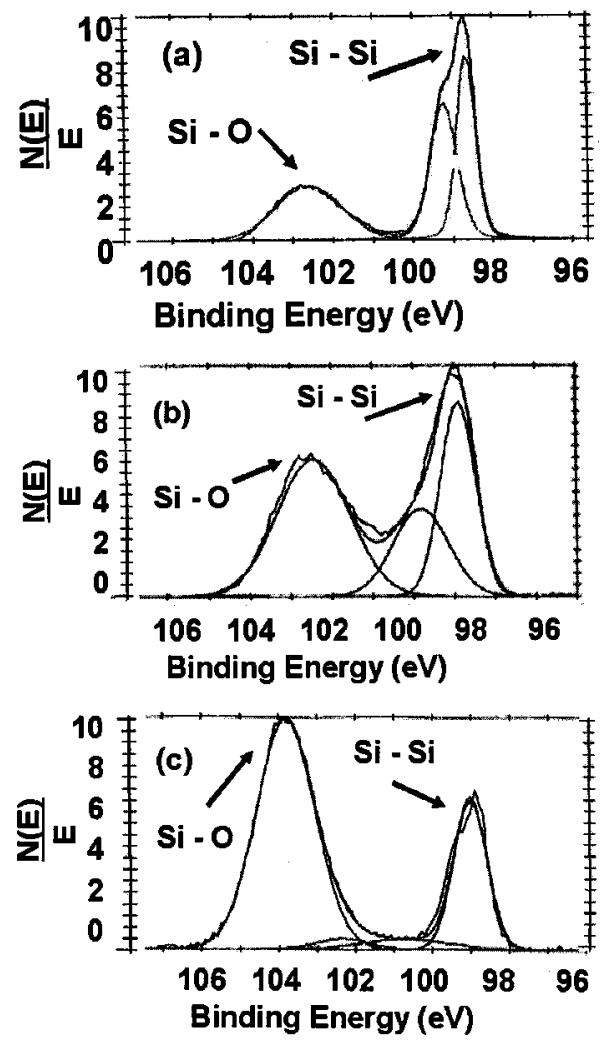

FIG. 11. Shallow take-off angle XPS analysis of a pre-GCIB Si with a native oxide (a), after a $7 \mathrm{kV} 1 \times 10^{15} \mathrm{ions} / \mathrm{cm}^{2}$ Ar-GCIB process (b), and after a $7 \mathrm{kV} 1 \times 10^{15}$ ions $/ \mathrm{cm}^{2} \mathrm{O}_{2}$-GCIB process (c) (see Ref. 24). The pre-GCIB Si surface (a) exhibits a surface roughness $R_{a}=0.9 \AA$. The 1 $\times 10^{15}$ ions $/ \mathrm{cm}^{2}, 7 \mathrm{kV}$ Ar-GCIB processed surface (b) yields a roughness $R_{a}=12 \AA$. The $1 \times 10^{15}$ ions $/ \mathrm{cm}^{2}, 7 \mathrm{kV} \mathrm{O}$-GCIB processed surface (c) yields a roughness $R_{a}=2.3 \AA$.

For the surface imaging of the high-energy individual Ar cluster impacts, the four-fold hillock and three-fold hillock formation may be explained through gas cluster kinetics and surface chemistry. The HRTEM, AFM, and MD simulations of this article suggest that the fundamental kinetic process incorporates an isotropic transfer of the cluster kinetic energy to the target that produces the symmetrical crater. The distribution of ejected material from the crater is portrayed in the AFM image of the hillock.

The hillock formations from individual cluster impacts show that they are the initial surface features of the GCIB smoothing process. For higher fluence GCIB processes, these hillocks eventually overlap by sheer number of crater impact events to provide full surface coverage. For higher (1 $\times 10^{15}$ ions $/ \mathrm{cm}^{2}$ ) fluence $\mathrm{Ar}$ and $\mathrm{O}_{2}$ GCIB processed Si surfaces with a native oxide, a continuous surface oxide formation of over $100 \AA$ is consistently observed by SE measurement and modeling, and x-ray photoemission spectroscopy (XPS). As shown in Fig. 11, XPS suggests that, for high fluence $\mathrm{Ar}$ GCIB processing on $\mathrm{Si}$, the resulting surface oxide is substoichiometric. ${ }^{24,25}$ For the case of high fluence $\mathrm{O}_{2}$ GCIB processing on $\mathrm{Si}$, XPS has indicated a more stoichiometric surface oxide, as evidenced by the stronger Si-O $2 p$ electron binding intensity and slight shift to $104 \mathrm{eV}$ as shown in Fig. 11. However, the precise composition of the full coverage surface layer is not determined. The approximate 
chemical composition of the individual hillocks may be inferred from the higher fluence GCIB surface SE and XPS observations. The hillocks are thus suggested to be a substoichiometric oxide in the case of Ar GCIB processing and a more stoichiometric oxide formation for the case of $\mathrm{O}_{2}$ GCIB processing.

The Ar cluster has enough energy to impact through the $\sim 20 \AA$ native surface oxide to the underlying Si crystal and eject $\mathrm{Si}$ from the substrate matrix. Cluster impacts provide for new exposure of $\mathrm{Si}$ surface atoms and their subsequent reactivity with the presence of oxygen. In the Ar-GCIB process case, the gas cluster itself does not provide a source of oxygen to react with the ejected silicon from the matrix (as in the case for oxygen-GCIB processes). Instead, adsorbed water from the surrounding surface area could provide the oxygen to form the surface hillock. Eventually, the stochastic overlay of the hillock material across the entire surface will provide, in the case of higher fluence Ar GCIB, a complete surface coverage of that substoichiometric oxide composition.

In the case of $\mathrm{O}_{2}$-GCIB processing, the $\mathrm{O}_{2}$ cluster immediately provides oxygen atoms for reaction with the silicon atoms of the newly exposed matrix. In this case, formation of a reactive product and an evaporation or ejection of the product from the surface takes place. For the oxygenGCIB process, the kinetically preferred (111) crystallographic sidewall formation appears to be a secondary factor in crater morphology to that of the reactive product formation. The immediate provision of oxygen to the silicon matrix provides an expedient method of reducing the overall energy of the system through oxygen-silicon bonding along the crater wall, and inducing the bowl shape of the crater as indicated by MD simulation. The surface hillocks formed by the $\mathrm{O}_{2}$ cluster impacts have not exhibited four-fold or threefold symmetry. Rather, these $\mathrm{O}_{2}$-GCIB individual impact hillocks have exhibited uniform coverage around and above the crater. The $\mathrm{O}_{2}$-GCIB hillocks are, in general, of much lower height and with larger horizontal spread than for $\mathrm{Ar}$ GCIB. Since the ejected material is fully reacted from the cluster source atoms, their trajectories may be slightly different from that of nonreacted silicon atoms that can bond with oxygen along their paths.

While high- and low-energy individual $\mathrm{O}_{2}$ cluster impacts into $\mathrm{Si}$ have not been previously reported, Gruber et $a l .{ }^{4}$ have observed an individual $\mathrm{CO}_{2}$ cluster impact into $\mathrm{Si}$ that also displays a rounded hillock surface. They have reported the hypervelocity impact will create a crater from the release of shock waves in the silicon and that the hillock is created by an elastic rebound of the compressed material. We further suggest that reactive chemistry from the oxygen in the cluster (or the surrounding atmosphere) plays a significant role in the ejected material distribution and may prevent the observation of symmetry.

The shape of the crater remains constant with energy, with the $3 \mathrm{kV}$ crater providing a shallow version of the 24 $\mathrm{kV}$ crater for both the $\mathrm{Ar}$ and $\mathrm{O}_{2}$ gas cluster cases. More important for commercial applications, the specific energy of the gas cluster processes provides some insight into the use of GCIB for smoothing surfaces. In starting to smooth a material surface with deep scratches, for example, the initial use of a higher-energy GCIB process will provide a faster etch rate and provide for a more uniform surface morphology frequency distribution across the surface. ${ }^{8,26}$ The use of a lower-energy GCIB as a final process step provides a smaller crater depth and hillock height for a smoother surface finish. The choice of cluster gas type (e.g., changing from argon to oxygen) may also provide a smoother surface due to a more rounded or shallow crater contour. The crater morphology approach is supported by the AFM measurements associated with the data of Fig. 11 for the Ar-GCIB and $\mathrm{O}_{2}$-GCIB higher fluence processes. The lower-energy $(7 \mathrm{kV}) 1 \times 10^{15}$ ions $/ \mathrm{cm}^{2}$ Ar-GCIB process yields an average surface roughness $\left(R_{a}\right) \sim 12 \AA$. For the $7 \mathrm{kV}$ and $1 \times 10^{15}$ ions $/ \mathrm{cm}^{2}$ fluence using an $\mathrm{O}_{2}$-GCIB process, the value is reduced to $R_{a} \sim 2 \AA$. The data indicate that the lower-energy gas clusters that provide a rounded crater are suitable for smoothing material surfaces. Alternately, higher-energy impacts resulting in a deeper crater are more effective for shallow etching of surfaces.

\section{CONCLUSION}

Further insight into the nature of single gas cluster impacts into a silicon surface has been provided. Experiment and simulation data for individual Ar gas cluster impacts into $\mathrm{Si}(100)$ with a native oxide reveal a crystallographic crater sidewall orientation and suggest a four-fold symmetric ejection of the silicon atoms to the surface. The presence of oxygen (adsorbed water, the $\mathrm{O}_{2}$ gas cluster itself) will provide for postimpact silicon-oxygen reaction. The ejected material forms hillocks over the crater. In the case of low fluence, high-energy Ar GCIB, these symmetric hillocks form over the native oxide to heights $>50 \AA$. AFM of the hillock surface shows (and MD simulation predicts) a fourfold symmetric hillock formation for the higher-energy ArGCIB individual impacts into $\mathrm{Si}(100)$ and a three-fold (sixfold) symmetry for the same energy Ar-GCIB individual impacts into $\mathrm{Si}(111)$. This is consistent with the density of atoms for the respective Si crystal orientations. The effective use of GCIB energy and chemistry for tailoring the roughness or smoothness of different material surfaces may be approached through the analysis of the crater depth, the crater contour, and the stochastic overlay of individual craters to form the finished material surface.

\section{ACKNOWLEDGEMENTS}

The authors gratefully acknowledge valuable discussions with John Hautala, Tom Tetreault, Yan Shao, Mike Mack, and Allen Kirkpatrick of Epion Corporation. The integral support of the USAF under Contract No. F33615-00-C-5404 is sincerely acknowledged. GCIB equipment and process work under DOC-NIST ATP award Contract No. 70NANB8H4011 is also appreciated.

\footnotetext{
${ }^{1}$ J. -H. Song, S. N. Kwon, D. -K. Choi, and W. -K. Choi, Nucl. Instrum. Methods Phys. Res. B 179, 568 (2001).

${ }^{2}$ I. Yamada, J. Matsuo, N. Toyoda, and A. Kirkpatrick, Mater. Sci. Eng., R. 34, 231 (2001).
} 
${ }^{3}$ P. von Blanckenhagen, A. Gruber, and J. Gspann, Nucl. Instrum. Methods Phys. Res. B 122, 322 (1997).

${ }^{4}$ A. Gruber and J. Gspann, J. Vac. Sci. Technol. B 15, 2362 (1997)

${ }^{5}$ I. Yamada, J. Matsuo, Z. Insepov, D. Takeuchi, M. Akizuki, and N. Toyoda, J. Vac. Sci. Technol. A 14, 781 (1996).

${ }^{6}$ H. Hsieh, R. S. Averback, H. Sellers, and C. P. Flynn, Phys. Rev. B 45, 4417 (1992).

${ }^{7}$ Z. Insepov and I. Yamada, Mater. Sci. Eng., A 217, 89 (1996).

${ }^{8}$ D. B. Fenner, R. P. Torti, L. P. Allen, N. Toyoda, A. R. Kirkpatrick, J. A. Greer, V. DiFilippo, and J. Hautala, Mater. Res. Soc. Symp. Proc. 585, 27 (2000).

${ }^{9}$ Z. Insepov, M. Sosnowski, and I. Yamada, Trans. Mater. Res. Soc. Jpn. 17, 111 (1994).

${ }^{10}$ I. Yamada and J. Matsuo, Mater. Res. Soc. Symp. Proc. 396, 149 (1996).

${ }^{11}$ I. Yamada and J. Matsuo, Mater. Res. Soc. Symp. Proc. 427, 265 (1996).

${ }^{12}$ D. B. Fenner, J. Hautala, L. P. Allen, T. G. Tetrault, A. Al-Jibouri, J. I. Budnick, and K. S. Jones, J. Vac. Sci. Technol. A 19, 1207 (2001).

${ }^{13}$ I. Yamada, W. L. Brown, J. A. Northby, and M. Sosnowski, Nucl. Instrum. Methods Phys. Res. B 82, 223 (1993).

${ }^{14}$ Z. Insepov and I. Yamada, Nucl. Instrum. Methods Phys. Res. B 99, 248 (1995).

${ }^{15}$ M. Moseler, J. Nordiek, and H. Haberland, Phys. Rev. B 56, 15439 (1997).

${ }^{16}$ Z. Insepov, M. Sosnowski, and I. Yamada, Nucl. Instrum. Methods Phys. Res. B 127, 269 (1997).
${ }^{17}$ Z. Insepov, R. Manory, J. Matsuo, and I. Yamada, Phys. Rev. B 105, 11605 (2000).

${ }^{18}$ Y. B. Zeldovich and Y. P. Raiser, Physics of Shock Waves and HighTemperature Hydrodynamic Phenomena (Academic, New York, 1967), p. 653.

${ }^{19}$ L. D. Landau and E. M. Lifshitz, Theory of Elasticity (Pergamon, Oxford, 1986).

${ }^{20}$ Handbook of Chemistry and Physics, edited by D. R. Lide (CRC Press, London, 1993), p. 4-1.

${ }^{21}$ H. Haberland, Clusters of Atoms and Molecules I (Springer, Berlin, 1995), Chap. 3.

${ }^{22}$ J. Farges, M. F. DeFeraudy, B. Raoult, and G. Torchet, Ber. Bunsenges. Phys. Chem. 88, 211 (1984).

${ }^{23}$ J. Won Lee and G. D. Stein, J. Phys. Chem. 91, 2450 (1987).

${ }^{24}$ L. P. Allen, K. S. Jones, C. Santeufemio, W. Brooks, D. B. Fenner, and J. Hautala, Proceedings of the Near Surface Effects Conference, 5-10 August 2001, Kodiak Island, Alaska, p. 16 (woodc@onr.navy.mil, ONR, Arlington, VA)

${ }^{25}$ L. P. Allen, D. B. Fenner, C. Santeufemio, W. Brooks, J. Hautala, and Y. Shao, in Proceedings of the SPIE International Symposium on Optical Science and Technology, 7-11 July 2002, Seattle, WA, Vol. 4806, p. 225.

${ }^{26}$ D. B. Fenner, D. W. Dean, V. DiFilippo, L. P. Allen, J. Hautala, and P. B. Mirkarimi, Mater. Res. Soc. Symp. Proc. 647, O5.2.1 (2001). 